\title{
Intraoperative parathyroid hormone assay during surgery for secondary hyperparathyroidism: is it time to give up the chase at the hormone?
}

\author{
Paolo Miccoli
}

Published online: 30 June 2012

(C) Springer Science+Business Media, LLC 2012

In the early 1990s, when George Irvin reported his first results about the use of a quick intraoperative parathyroid hormone assay (ioPTHa) during surgery for primary hyperparathyroidism (PHPT), this innovative instrument appeared to be an extremely promising way to provide surgeons with reliable guidance while performing a parathyroidectomy for PHPT [1]. In fact, the method had an acceptable certainty of confirming in most patients that all of the hyperfunctioning parathyroid tissue in the neck had been removed, to the extent of defining it as a "biochemical frozen section." Some of the limits of the technique became evident quite soon, because a limited but significant number of false-positive and false-negative results seemed to be unavoidable in every series that was reported, adding some uncertainty about the real effectiveness of the tool [2].

The main problem was how to determine the cutoff for the PTH drop rate and the timing of the blood sample to be tested. The choice of these two data can heavily condition the balance between a false-positive and a false-negative result [3]. These limits can influence even more the outcomes in patients with secondary HPT (SHPT) and tertiary HPT, where the cutoff seems to be more difficult to determine, for several reasons [4-6].

Correctly, Conzo et al. [7] quoted several proposed cutoff values in their report as a percentage of the initial value or as an absolute value. Also, the timing varied greatly from series to series. The choice by these authors to choose a 20-min cutoff seems reasonable. Even though the half-life of this hormone is limited to very few minutes, its

P. Miccoli $(\bowtie)$

Department of Surgery, University of Pisa, Via Paradisa 2,

56124 Pisa, Italy

e-mail: p.miccoli@med.unipi.it values at the beginning of the operation are much higher than in PHPT, and because of the manipulation, which involves all of the glands, last much longer. Thus, considering a time consistently longer than $10 \mathrm{~min}$ should guarantee better results. For the same reason, it is more important to establish a percentage of the PTH drop with respect to the initial value rather than its absolute value. In fact, if all the parathyroid tissue were removed, PTH should be, presumably, 0; but this never happens.

Nevertheless, a really "total" parathyroidectomy is mandatory, and by then, the use of ioPTHa could significantly modify the surgical strategy. It is well known, indeed, that removal of four enlarged parathyroid glands is not sufficient to consider the operation successful: a fifth and even a sixth gland can be present in these patients in significantly higher percentages than in patients affected by PHPT [4], thus inducing the surgeon to continue the neck exploration. In these patients, an ablation of the upper horns of the thymus on both sides would be highly advisable.

However, even if the expected drop does not occur after $20 \mathrm{~min}$, this does not necessarily mean that cure has not been achieved, because the PTH level might decrease much later than 20 min after surgery. Because a supernumerary gland might be in the mediastinum, an extensive and time-consuming neck exploration is sometimes unreasonable. For similar reasons, most surgeons do not advocate the use of sophisticated and costly preoperative imaging studies, and in any event, these cannot substitute for the experience of a surgeon with a high volume of parathyroidectomies [8]. Despite these limits, the use of ioPTHa might help an experienced surgeon in tailoring his or her operative strategy.

The possible prognostic value of ioPTHa to predict a possible hypoparathyroidism after surgery, which comes 
much later, constitutes a different problem. Its real value is still to be exactly determined, because the results that have been reported are arguably uniform. Its cost-effectiveness probably does not justify the investment, because the costs seem not to match the real advantages.

Although several models have been proposed to predict possible hypoparathyroidism after parathyroidectomy or total thyroidectomy, none seem to offer a prognostic certitude to single out the patients that can be discharged on the same day of surgery without any calcium and vitamin D supplementation and possibly keeping the other patients in the hospital or giving them an immediate substitutive therapy. This would be particularly important in centers where thyroid and parathyroid surgery is mainly performed on an outpatient basis.

This problem is probably less important when dealing with SHPT because these patients are hospitalized longer than those undergoing surgery for PHPT or thyroid disease. According to Roshan et al. [6], in the same sample of patients where the biochemical resolution of SHPT was correctly predicted by the use of intraoperative PTH assay, the same failed to predict a persistent hypoparathyroidism, thus revealing an arguable efficacy. All the same, many other studies involving this aspect are not at all concordant in the interpretation of their results and the possible advantages of the tool in patients with SHPT $[9,10]$, whereas the results appear far more concordant for the treatment of patients with tertiary HPT after a successful renal transplantation.

We might assume that the risk of HPT should be carefully avoided in patients presenting a SHPT, in particular if waiting for a kidney transplant; however, the ioPTHa can be of some use in predicting the success of the surgical operation, and perhaps even in helping surgeons to tailor their strategy, but is of poor use in predicting hypoparathyroidism after surgery [10].

Conflict of interest The author declares that there is no conflict of interest with regard to this article.

\section{References}

1. G.L.D. Irvin, V.D. Dembrow, D.L. Prudhomme, Clinical usefulness of an intraoperative "quick parathyroid hormone" assay. Surgery 114, 1019-1022 (1993)

2. L.F. Morris, K. Zanocco, P.H. Ituarte, K. Ro, Q.Y. Duh, C. Sturgeon, M.W. Yeh, The value of intraoperative parathyroid hormone monitoring in localized primary hyperparathyroidism: a cost analysis. Ann. Surg. Oncol. 17(3), 679-685 (2010)

3. D.M. Carneiro, C.C. Solorzano, M.C. Nader, M. Ramirez, G.L. Irvin 3rd, Comparison of intraoperative iPTH assay (QPTH) criteria in guiding parathyroidectomy: which criterion is the most accurate? Surgery 134(6), 973-979 (2003)

4. J. Lokey, F. Pattou, A. Mondragon-Sanchez, M. Minuto, B. Mullineris, F. Wambergue, P. Foissac-Geroux, C. Noel, H.L. de Sagazan, P. VanHille, C.A. Proye, Intraoperative decay profile of intact (1-84) parathyroid hormone in surgery for renal hyperparathyroidism-a consecutive series of 80 patients. Surgery 128(6), 1029-1034 (2000)

5. M. Barczyński, S. Cichoń, A. Konturek, W. Cichoń, A randomised study on a new cost-effective algorithm of quick intraoperative intact parathyroid hormone assay in secondary hyperparathyroidism. Langenbecks Arch. Surg. 390(2), 121-127 (2005)

6. A. Roshan, B. Kamath, S. Roberts, S.L. Atkin, R.J. England, Intra-operative parathyroid hormone monitoring in secondary hyperparathyroidism: is it useful? Clin. Otolaryngol. 31, 198-203 (2006)

7. G. Conzo, A. Perna, N. Avenia, R.M. De Santo, C. Della Pietra, A. Palazzo, A.A. Sinisi, F. Stanzione, L. Santini, Evaluation of the 'putative' role of intraoperative intact parathyroid hormone assay during parathyroidectomy for secondary hyperparathyroidism. A retrospective study on 35 consecutive patients: intraoperative $\mathrm{iPTH}$ assay during parathyroidectomy. Endocrine (2012). doi:10.1007/s12020-012-9648-5

8. G. Gasparri, M. Camandona, U. Bertoldo et al., The usefulness of preoperative dual-phase $99 \mathrm{~m}$ Tc MIBI-scintigraphy and IO-PTH assay in the treatment of secondary and tertiary hyperparathyroidism. Ann. Surg. 250(6), 868-871 (2009)

9. K. Kaczirek, P. Riss, G. Wunderer, G. Prager, R. Asari, C. Scheuba, C. Bieglmayer, B. Niederle, Quick PTH assay cannot predict incomplete parathyroidectomy in patients with renal hyperparathyroidism. Surgery 137(4), 431-435 (2005)

10. B.P. Müller-Stich, M. Brändle, I. Binet, R. Warschkow, J. Lange, T. Clerici, To autotransplant simultaneously or not-can intraoperative parathyroid hormone monitoring reliably predict early postoperative parathyroid hormone levels after total parathyroidectomy for hyperplasia? Surgery 142(1), 47-56 (2007) 